Thora Tenbrink*

\title{
Linguistic spatial reference systems across domains: How people talk about space in sailing, dancing, and other specialist areas
}

https://doi.org/10.1515/lingvan-2020-0041

\begin{abstract}
Spatial reference systems have been investigated across many different languages and cultures, partly with the explicit aim of identifying preferred ways of thinking and talking about space in a particular culture. This paper addresses variability within a given language (and culture) by looking at diversity across everyday specialist domains. Wherever a domain requires people to interact with space in a specific way, conventions for thinking and talking about space arise that are far less common outside those domains. For instance, in sailing it is almost impossible to talk about 'forward movement', due to the various forces acting on the boat; these require the sailor to calculate a useful course relative to the goal direction. Based on a range of examples, this paper explores customary ways of talking about space across various domains, and highlights the underlying spatial-conceptual reference systems. This demonstrates how different situational domains call for different reference systems, contrary to beliefs that entire cultures can be associated with stable preferences for a specific reference system.
\end{abstract}

Keywords: variability, spatial reference systems, specialist domains, context, sociotopography

\section{Introduction}

How we think about spatial relationships in our environment depends on who we are, as well as on features of the environment. In Germany, where I grew up, it is common to associate North with coast, and South with mountains. These prominent geographical features affect how the country's spatial structure is conceived. The orthogonal direction of East and West, on the other hand, is collectively associated with the former political border within the country, even though this division officially disappeared decades ago. Other countries have different features, and the geographical and cultural or political aspects will affect spatial concepts in different ways (Palmer 2015).

Observations such as these may have inspired the host of research available today that demonstrates the diversity of spatial reference systems between cultures and languages, perhaps most famously in the extensive work of Stephen Levinson (e.g., 2003) and his co-authors in associated projects. Recognition of cross-cultural diversity in spatial thinking is especially important in light of a history of linguistic enquiry that has been extremely biased towards English speaking countries throughout most of its existence.

At the same time, this generally laudable orientation towards other cultures seems to be associated with what might be regarded as a cognitive simplification process, a way of coping with the unfathomable complexity of the world's diversity (following Gigerenzer 2000): a perhaps not-so-laudable tendency to describe entire cultures as if they were uniform. Consider my suggestive example at the beginning of this section: Germans tend to conceive of the country in terms of political areas to the East and West, and in terms of geographical features to the North and South. Well, do they? All of them? And who are "they", exactly? Under which circumstances are these directional distinctions relevant? What other conceptions of space can be found in Germany, and under what circumstances are those relevant? Once we start

*Corresponding author: Thora Tenbrink, Bangor University, Bangor, Wales, UK, E-mail: t.tenbrink@bangor.ac.uk. https://orcid. org/0000-0002-7986-1254

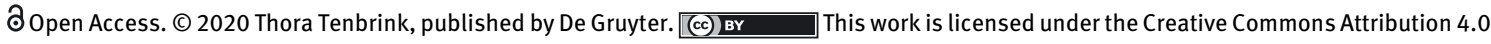
International License. 
thinking along these lines, it is very clear that any generalised statement of this kind is bound to be a rather crude simplification. In fact, results increasingly emerge that demonstrate considerable diversity within various cultures (Palmer et al. 2018).

In this paper, I will return to the Western-centred point of view that served as a starting point for the exploration of spatial reference systems, and examine the diversity of spatial concepts and reference systems that can be found within a range of specialist areas in English-speaking communities. Based on concrete examples taken from domains such as sailing, dancing, and horse riding, I aim to demonstrate that spatial concepts are determined by relevance to a specific context, which includes not only language and wider culture but also the function and purpose of the spatial conceptualisation itself, situated in a certain spatial layout. This complements previous insights into the various kinds of influence contributed by the environment's topography (Palmer 2015), culture-engrained cognition (Levinson 2003), task specificity (Bohnemeyer 2011), and linguistic factors in their interplay with patterns of language use (Palmer et al. 2018).

\section{Spatial language and cognition: A brief overview}

Several decades of research in spatial language and cognition in English and across cultures have made available a host of insights that cannot be done justice to in a short section. Instead, my aim here is to provide a few starting points for the considerations in the next sections, and to review briefly the kinds of aspects that have been researched in great depth.

Much of the literature on spatial language and cognition falls into one of two fields: either spatial object reference, i.e., patterns of how an object's location is frequently described in everyday life relative to another object, or route descriptions that highlight how we tend to conceptualise and verbalise spatial navigation to a destination. These two fields capture recurrent aspects of common everyday interactions in spatial environments generically to a considerable extent: we frequently locate (or move) objects, or move (or locate) ourselves in space.

For spatial object reference primarily, Levinson (2003) suggested a three-way classification of linguistic spatial reference frames often used to describe where one object (the 'locatum', also called 'figure' or 'referent') is located in relation to another object (the 'relatum', also called 'ground' or 'reference object'). In intrinsic reference systems, the locatum is positioned relative to intrinsic features of the relatum, e.g., Peter's front in The table is in front of Peter. In relative reference systems, the locatum is positioned relative to the relatum from another position or point of view, as in The table is in front of the wall from my point of view. Finally, absolute reference systems make use of ubiquitous environmental features such as compass directions, e.g., The house is to the North of the lake. This classification has been used widely to describe cultural patterns and preferences (Senft 2001), relationships between language and cognition when considering spatial relations (Levinson 2003), conceptual diversity across different configurations in space and time including motion events (Tenbrink 2011), and more. Although linguistic descriptions tend to schematise spatial objects and their relative locations, abstracting from details of shape and exact geometric details or metrics (Landau and Jackendoff 1993; Talmy 2000), object features do affect spatial description in a range of ways (van der Zee and Eshuis 2003), including how reference frames are applied.

Spatial reference systems are also frequently used to describe navigation concepts, for instance to examine switches in perspective (Tversky et al. 1999). Due to the differences between moving in environments as opposed to locating objects, in this context it is more common to distinguish between route and survey perspectives (Taylor and Tversky 1992) or egocentric and allocentric reference frames. The latter is a distinction mainly found in cognitive science (e.g., Burgess 2006), independent of language. In the context of route navigation, both categorisations have in common that they distinguish between an observer's point of view, representing the navigator's perception (as in turn right), and a top-down point of view of the kind found in a map, where spatial relations are described independent of a specific position. Further aspects addressed in the area of navigation and linguistic representation concern landmarks (Caduff and Timpf 2008), hierarchical concepts (Klippel et al. 2009), wayfinding strategies (Hölscher et al. 2009) and much more (Denis 2018). 


\section{Sailing}

Sailing is a popular modern sport that, while not accessible to everyone due to a number of requirements (such as physical, geographical, or infrastructural), spans a wide variety of engagement types. While enthusiastic dinghy sailors will be focused on efficiency, speed, and competition within a predefined and often small area on the water, leisure yacht sailors may be more interested in navigating quietly to a chosen location further away. Whatever the level and purpose of engagement, however, most sailors will at some point come across challenges resembling those sketched in the previous section: objects (or people, or places) will need to be located, and goals need to be reached by navigating the available space.

Tenbrink and Dylla (2017) explored these challenges in relation to Levinson's (2003) categorisation of spatial reference systems. This work was driven initially by the simple personal observation that sailors rarely use the word forward in the context of sailing. Why would they avoid this term? According to Tenbrink (2011), the notion of forward movement normally involves a relocation of a person to a position in front of the current position, using the intrinsic reference system of that person which is based on the person's current orientation. When walking forward, the person's current perspective (their view direction) typically corresponds to the direction of movement. However, during sailing, this normally straightforward notion of perspective is far more complicated (Tenbrink and Dylla 2017). Especially in a dinghy, it is fairly likely that the sailor isn't actually looking in the direction of movement. Moreover, multiple directional forces offer alternative perspectives, such as the boat's intrinsic orientation, the boat's current direction of movement, the wind direction, or the direction to a visible goal of navigation.

This contrasts with most other modes of transport in everyday life. When driving a car (or a bike, or a bus), we can easily refer to forward movement because the driver's view direction will normally correspond to the vehicle's direction of movement, and no further forces are involved. This is also the case for some kinds of water sports like kayaking or canoeing, except that the water's movement (current or tide, or wind forces affecting the surface) may affect the sense of forward movement. In rowing, matters are more complicated because the rower sits with their back to the front side (bow) of the boat. Which direction is forward in a rowing boat - and are rowers actually going backward? If this is not straightforward to answer, this might illustrate the problem with forward motion during sailing. Sailors could be anywhere in the boat, looking in any direction - and the boat's orientation and movement direction will be so much affected by the wind's force on the sails that the concept forward appears to lose its meaning. Sailors can rarely head directly to their goal, as they need to orient the boat relative to the wind - and then they typically need to change direction (tack or gybe) several times before reaching the goal.

Instead of referring to a forward direction, therefore, sailors talk about course made good - making progress towards an intended goal; or they use more specific concepts and jargon specific to sailing: port and starboard, windward, leeward, into the wind, and so on. Clearly, everyday language is not felt to be sufficient on the local level of orienting in a specific direction (Tenbrink and Dylla 2017).

On a more global level, navigation concepts for sailing can become even more complicated. While no specific training (other than relevant everyday experience) is typically needed in order to find one's way through a street network with the help of maps or navigation systems, yacht sailors are expected to undergo courses (such as those offered by the British Royal Yachting Association) at various levels before they are allowed to hire a boat. Those courses include skills such as interpreting buoyage, reading charts, plotting a course to a goal, collision regulations, position fixing, and much more. Such skills are spatial to a high degree, and they involve various reference systems to assist navigation (e.g., compass directions, the Global Positioning System (GPS), or assessing one's course relative to the sun and the stars).

Compare this to the knowledge needed for navigation in everyday contexts. The kinds of typical spatial elements and concepts that tend to be taken into account when navigating to a goal in a street network are fairly well understood by now, based on extensive research over the last decades (Denis 2018). As mentioned in the previous section, routes in such settings can be described on the basis of route or survey perspectives, both of which can be straightforwardly defined and identified. Wayfinders will normally rely on a range of spatial 
elements that are relevant for them, such as decision points, route segments, landmarks, reorientations, movement directions, regions, and distance concepts (Tenbrink 2012). All of these are fundamentally different in nature during sailing.

Take decision points, for example: In a street network, decision points are intersections where the navigator can turn right or left or carry on straight. These are frequently referred to by describing the intersections themselves or by reference to landmarks (Denis 1997). Outside street networks, in open natural landscapes, the notion of decision points is more complex; in a mountaineering context, for instance, one may find references to where the snow starts as part of the expression of directional decisions (Egorova et al. 2015). However, on the water, there are hardly any local landmarks except for the occasional buoy. Sailors don't turn left or right even there, or only rarely; instead, they may follow the buoys or keep them to starboard or port while heading into a harbour.

Sailors may also orient towards distant landmarks to find their bearing, which is more similar to phenomena found in everyday navigation contexts (Klippel and Winter 2005). Much more frequently, however, GPS information would be used for positioning and compass directions for orienting purposes. Both of these are available in other contexts too, but used far less extensively due to the availability of more accessible, less abstract types of spatial information. On the water, when traversing larger distances, there is very little to orient towards - but sailors have always made use of whatever there is available, using sun and the stars if required (Lewis 1974).

As spatial environments and cultural habits differ widely with respect to water, cultures will widely differ in the associated communication (except for being inspired by or learning from each other). While different from spatial communication when navigating on land or referring to objects nearby, sailing communication will reflect the cognitive and communicative principles of the cultures involved its own way. The famous insights on Polynesian navigation skills are a case in point (Finney 1994).

\section{Dancing}

Sailing is not the only everyday domain in which conventional ways of referring to space appear to be insufficient. Challenges occur whenever movement in space is more complicated and departs from what most people do in their daily life. Dancers, for instance, change their posture and direction of view rather more frequently than people usually do. Spatial awareness is therefore seen as integral to dance, with extensive practice required to develop the necessary skills of both exteroceptive (perception-based) and interoceptive (sensorimotor) types of awareness. ${ }^{1}$ References to the importance of space (as well as time) are ubiquitous across online professional sources as well as academic literature on dance. Some authors even develop their own terminology to describe the specificity of motion in this art: "Dancing is sortally generating movings by bodily motions and posings by bodily pauses.” (Beardsley 1982: 32). Poetic terminology such as 'the body's flow in space' (Stewart 1998: 46) is equally abundant in this context, as are highly complex descriptions of intricate movements.

The conventionally used static or dynamic reference systems of the kind described in section 2 seem fairly simple in comparison. Consider the following example of specific movements (Stewart 1998: 47): “Nakajima's trunk tilts towards forward-high and twists to the right from the waist. The right arm simultaneously extends to left-side-middle in front of the left arm which remains flexed as it inclines towards left-forward-low. She faces forward-middle". While the underlying conceptual reference system used here appears to be consistently intrinsic (using the dancer's own perspective), the frequent directional combinations are extraordinary.

Rather than using this intricate kind of description, dancers would often refer to complex patterns of movement as figures or sequences of motion, combining elements that are typically beyond a non-dancer's everyday repertory, such as skipping, sliding, bending, swinging, tilting, twisting and the like. Such pre-defined

1 https://ballroomdanceplanet.com/spatial-patterns-in-dance/ 
patterns or 'gestures' can then, as such, serve as reference frames for movements to be aligned with patterns in the music: "basic gestures are spatiotemporal shapes of movement trajectories of body parts that function as frames of reference for the guidance of the coupling of dance and music" (Naveda and Leman 2010: 94). Formal approaches have aimed to capture such structures and pattern-based meanings in dance movements for semantic querying purposes (Ramadoss and Rajkumar 2006). This ties in with a long-standing recognition that dance can be regarded as a form of language (Wigman 1966), with strong expressive and representative functions reflected through controlled, creative motion in space and time.

While traditional forms of dance, as such, therefore provide a promising area for spatial language research, an even more spatially extraordinary form of dance has emerged in recent years, called vertical dance (Lawrence 2010). In this dance form, the dancer uses a vertical plane, typically a wall, as their 'dance floor', suspended in the air by a waist harness. Clearly, this opens up fundamentally new ways of experiencing space. Lawrence's description of this experience spells this out wonderfully, justifying a full quote here:

"The vertical dancer's spatial frame of reference is therefore contingent on the movement she is performing. For example, when she is upside-down from the spectator's point of view, that is, with the crown of her head pointing toward the ground, it is the downward relationship between the ground and the crown of her head that is the most palpable as she is acutely aware that the ground is below her (standard cross of axis). When she faces the side, in order to combat the urge to return to verticality, she perceives the horizon to be the upward coordinate (body cross of axis). In this orientation, she senses the audience, who perceive themselves to be below her, as diagonally above her. Thus, her elevated situation in height turns out, in certain positions and situated within particular frames of reference, to be one of depth.” (Lawrence 2010: 56).

The main challenge, it seems, consists of the distortion of the canonical upright direction, which fairly consistently determines our standard ways of referring to the spatial world. Because of our permanent orientation to gravity, we can typically ignore the vertical direction altogether (Herskovits 1986). Perhaps as a consequence, relative reference frames (as described in section 2) do not appear to be available in the vertical dimension (Tenbrink 2007). Gravity serves as an anchor for an absolute reference frame, and the perceptions described in Lawrence's quote above are clearly intrinsic. However, a relative reference frame would mean relating two objects or locations to each other from a person's non-canonically oriented point of view rather than gravity, which seems not to be possible.

The fact that speakers normally rely so much on a normal upright orientation clearly hampers communication in a vertical dance context. References to up, forward, to the right and the like can be ambiguous even in ordinary situations, but with the speaker (or addressee) hanging upside down, further options for interpretation arise, rendering communication extremely challenging (Lawrence, pers. comm.). Speakers will thus aim to avoid these terms, referring to specific sides in the environment instead as in towards the floor/wall/audience. This strikingly parallels the avoidance of forward during sailing (see section 3).

\section{Further specialist areas}

The two domains explored so far are special with respect to spatial communication, but in this they are not extraordinary. Surely any specialist domain that involves moving in non-standard ways in space will come with its own spatial communication challenges - and strategies to tackle those. In horse riding, for example, the non-standard spatial communication challenges in this area comprise talking about gaits, biomechanics, using and changing reins, balance, riding a particular path or course in an arena, referring to directions in an open-space riding scenario, and more. For instance, understanding a horse's locomotion presupposes conceptualising a body that has a far more prominent sagittal (front-back) axis than humans do. On this basis, a wide range of translations and rotations can be performed, posing diverse challenges to the harmonious joint movement of two morphologically extremely different bodies across different gaits (Clayton and Hobbs 2017). In this context, the concept of a diagonal becomes rather more prominent than in ordinary everyday life, due to the horse's anatomy and movement patterns (Roepstorff et al. 2009). 
When riding in a circle or changing directions, horse riders talk about inside and outside as this can be applied generically (independent of direction). These spatial aspects (which are not so frequently applied in everyday life) are clearly more relevant in this context than left and right, as direction turns in horse riding involve changing the tension on the rein as well as pressure of the leg along with the bend of the horse. Consider the following spatial description of these effects: “Typically you ride 'inside leg to outside rein' which means you use your inside leg to push your horse's barrel toward the wall and maintain forward motion, while the outside rein is in steady contact to keep the horse's shoulders straight or control the size of the figure, so that your horse stays on track instead of falling away from the wall or figure onto their inside shoulder. When you change directions you change which rein is thus supporting outside rein, hence the name 'change of rein'. Despite the fact that communication thus downplays the human lateral asymmetry, humans nevertheless tend to apply somewhat more tension on the right-side rein (Eisersiö et al. 2015).

Further spatial specialist domains could be explored here - skiing downhill on a route-less mountain, juggling objects in free air, flying and navigating airplanes, diving in the deep sea, caving and potholing below the ground, designing built environments, three-dimensional art, navigating in outer space where gravity no longer determines verticality - the list could be extended infinitely as each domain offers extraordinary ways of dealing with space. Very few of these domains, if any, have been explored in any depth from a linguistic point of view, aiming to capture the spatial concepts and reference frames that emerge in each domain.

And further insights still can be gained by looking beyond spatial expert domains, into non-spatial domains that employ transferred spatial language to express concepts. Spatial language, as such, has been discussed at length with respect to such transferred meanings (e.g., Tyler and Evans 2003) - but the domains where such meanings are widely applied have received far less attention. To exemplify this point, consider the domain of music. Music is represented spatially, both in notation through spatially arranged black dots on a grid, and in conducting through systematic hand and arm movements representing tempo, rhythm, volume, expression and more. Paralleling this, music is also conceptualised spatially (Eitan and Granot 2006): changes in pitch are associated with changes in spatial verticality, pitch rise with approaching motion, and loudness changes with changes in distance. The language of music, in consequence, is abundant with metaphorical (or transferred) spatial references (Johnson and Larson 2003). However, the ways in which musical metaphors describe 'space' in this abstract domain clearly differ fundamentally from the purely spatial reference frames used for navigation and object reference, as they - like spatial specialist domains - represent very specific kinds of conceptualisations.

\section{Discussion and conclusion}

One of the questions frequently asked in spatial language research concerns the preference of a specific spatial reference system within a particular culture or language. Do speakers of English prefer intrinsic, relative, or absolute reference systems? Over the past decades, various arguments and empirical findings have been brought forward to claim one or the other (e.g., Carroll and von Stutterheim 1993 observed a preference for intrinsic reference systems in English as opposed to relative in German; Carlson-Radvansky and Radvansky 1996 argued that this might depend on functional relations between objects; and research by Levinson 2003 generally supports a relative reference system preference in English speaking cultures). However, it has been observed that a clear differentiation is very difficult to achieve in natural discourse situations due to multiple ambiguities (Tenbrink et al. 2011), hampering the identification of a generic preference within a language or culture.

In this paper, I have not attempted to make any claims along these lines, but rather aimed to highlight and illustrate the extremely wide variety of situations in which spatial language is used even within one language and culture. Of these, only a very small subset has been investigated concerning spatial reference frame use.

2 http://www.lessonsintr.com/2016/03/02/change-of-rein/ 
In the case of the sailing domain, for instance, Tenbrink and Dylla (2017) did not observe a preference for a particular reference frame but rather an avoidance of certain everyday terminology. Similarly, other specialist domains in which space is involved in non-standard ways call for distinct strategies of talking about spatial relationships and movements, where the existing classifications and insights concerning reference frames may not be sufficient as explanatory frameworks. Clearly, further research is required to explore the complex concepts and their representation in the English language more systematically, in order to account for the diversity of spatial concepts and reference frames comprehensively.

If English offers such a wide diversity, what does this imply for other languages and cultures around the world? The conclusion, I hope, should be relatively clear at this point: We can identify native speakers' preferences within a specific situation - introduced and controlled by the researchers, as is common practice in psycholinguistic studies - and we can compare these preferences between different groups of speakers with different linguistic backgrounds (as has been done, very insightfully, in many projects such as those described in Levinson 2003). We can also identify abilities and reference frame patterns that exist in one culture but not in another - take, for instance, the impressive ability of speakers of Yucatec Maya to point to and talk about absolute directions in space without the help of a compass or other visual aid (Le Guen 2011). However, we should be very careful in making claims about a culture's general preference. As demonstrated in Palmer et al. (2018), this kind of claim will never do justice to the wide diversity of activities in space (and beyond, using spatial metaphors) undertaken by humans around the globe. This diversity exists within language communities and even within individual speakers, depending on the specific domain of activity that are engaged in. Therefore, a broad generalisation about spatial referential strategy preferences at an entire language-community level does not capture the complexity of spatial reference, in line with a sociotopographic approach (Palmer et al. 2017).

Crucially, what is taken to be general and widely used in British, American or Australian English might not correspond to human habits in other cultures. For these variants of English, arguably generic everyday situations such as simple object localisations and route descriptions have been explored in great depth, but other domains have been largely ignored. The extent to which our insights about spatial reference in other cultures is actually based on their generic everyday situations will determine to what extent any generalised comparisons between cultures and languages may be justified. Clearly, studies of other languages are most valuable where they succeed in capturing frequent patterns of everyday life - but this may conflict with the traditional (and well-motivated) goal of using identical controlled experimental scenarios to allow for direct comparisons between cultures.

More specialised domains induce further complexity, even where there are parallels. Sailing in British waters is very different from sailing in Polynesia (Lewis 1974), and dance rituals in indigenous cultures fundamentally differ from Western dance performances (Bishop 2009). Spelling out relevant variability in spatial communication in non-everyday domains highlights variation in thinking about space around the globe, even though direct comparisons or claims about generic cultural preferences may remain out of reach.

\section{References}

Beardsley, Monroe C. 1982. What is going on in a dance? Dance Research Journal 15(1). 31-36.

Bishop, Joyce M. 2009. “Those who gather in": An indigenous ritual dance in the context of contemporary Mexican transnationalism. Journal of American Folklore 122(486).391-413.

Bohnemeyer, Jürgen. 2011. Spatial frames of reference in Yucatec: Referential promiscuity and task-specificity. Language Sciences 33. 892-914.

Burgess, Neil. 2006. Spatial memory: How egocentric and allocentric combine. Trends in Cognitive Sciences 10(12). 551-557.

Caduff, David \& Sabine Timpf. 2008. On the assessment of landmark salience for human navigation. Cognitive Processing 9(4). 249-267.

Carlson-Radvansky, Laura A. \& Gabriel A. Radvansky. 1996. The influence of functional relations on spatial term selection. Psychological Science 7. 56-60.

Carroll, Mary \& Christiane von Stutterheim. 1993. The representation of spatial configurations in English and German and the grammatical structure of locative and anaphoric expressions. Linguistics 31. 1011-1041. 
Clayton, Hilary M. \& Sarah-Jane Hobbs. 2017. The role of biomechanical analysis of horse and rider in equitation science. Applied Animal Behaviour Science 190. 123-132.

Denis, Michel. 1997. The description of routes: A cognitive approach to the production of spatial discourse. Cahiers de Psychologie Cognitive 16(4). 409-458.

Denis, Michel. 2018. Space and spatial cognition: A multidisciplinary perspective. London, New York: Routledge.

Egorova, Ekaterina, Thora Tenbrink \& Purves Ross. 2015. Where snow is a landmark: Route direction elements in alpine contexts. In Irina Fabrikant Sara, Raubal Martin, Bertolotto Michela, Davies Clare, Freundschuh Scott \& Bell Scott (eds.), Spatial information theory, 175-195. Berlin: Springer.

Eisersiö, Marie, Marie Rhodin, Lars Roepstorff \& Agneta Egenvall. 2015. Rein tension in 8 professional riders during regular training sessions. Journal of Veterinary Behavior 10(5). 419-426.

Eitan, Zohar \& Roni Y. Granot. 2006. How music moves: Musical parameters and listeners' images of motion. Music Perception 23(3). 221-248.

Finney, Ben R. 1994. Voyage of rediscovery: A cultural odyssey through Polynesia. Los Angeles: University of California Press. Gigerenzer, Gerd. 2000. Adaptive thinking: Rationality in the real world. Oxford: Oxford University Press, USA.

Herskovits, Annette. 1986. Language and spatial cognition. Cambridge: Cambridge University Press.

Hölscher, Christoph, Simon J. Büchner, Tobias Meilinger \& Gerhard Strube. 2009. Adaptivity of wayfinding strategies in a multibuilding ensemble: The effects of spatial structure, task requirements, and metric information. Journal of Environmental Psychology 29(2). 208-219.

Johnson, Mark L. \& Steve Larson. 2003. "Something in the way she moves" - metaphors of musical motion. Metaphor \& Symbol 18(2). 63-84.

Klippel, Alexander, Stefan Hansen, Kai-Florian Richter \& Stephan Winter. 2009. Urban granularities-a data structure for cognitively ergonomic route directions. Geolnformatica 13(2). 223-247.

Klippel, Alexander \& Stephan Winter. 2005. Structural salience of landmarks for route directions. In Anthony G. Cohn \& David M. Mark (eds.), COSIT 2005: International Conference on Spatial Information Theory. Ellicottville, NY, USA, September 1418, 2005, Proceedings, 347-362. Berlin: Springer.

Landau, Barbara \& Jackendoff Ray. 1993. "What" and "where" in spatial language and spatial cognition. Behavioral and Brain Sciences 16. 217-265.

Lawrence, Kate. 2010. Hanging from knowledge: Vertical dance as spatial fieldwork. Performance Research 15(4). 49-58.

Le Guen, Olivier. 2011. Speech and gesture in spatial language and cognition among the Yucatec Mayas. Cognitive Science 35(5). 905-938.

Levinson, Stephen C. 2003. Space in language and cognition. Cambridge University Press.

Lewis, David. 1974. Voyaging stars: Aspects of polynesian and Micronesian astronomy. Philosophical Transactions of the Royal Society of London. Series A, Mathematical and Physical Sciences 276(1257). 133-148.

Naveda, Luiz \& Marc Leman. 2010. The spatiotemporal representation of dance and music gestures using topological gesture analysis (TGA). Music Perception: An Interdisciplinary Journal 28(1). 93-111.

Palmer, Bill. 2015. Topography in language: Absolute frame of reference and the topographic correspondence hypothesis. In Rik De Busser \& Randy J. LaPolla (eds.), Language structure and environment: Social, cultural and natural factors, 179-226. Amsterdam: John Benjamins Publishing Company.

Palmer, Bill, Jonathon Lum, Jonathan Schlossberg \& Alice Gaby. 2017. How does the environment shape spatial language? Evidence for sociotopography. Linguistic Typology 21(3). 457-491.

Palmer, Bill, Alice Gaby, Jonathon Lum \& Jonathan Schlossberg. 2018. Diversity in spatial language within communities: The interplay of culture, language and landscape in representations of space. In 10th International Conference on Geographic Information Science (GIScience 2018). Schloss Dagstuhl-Leibniz-Zentrum fuer Informatik, Article No. 53. 53:1-53:8.

Ramadoss, Balakrishnan \& Rajkumar Kannan. 2006. Modelling and querying the expressive semantics of dance videos. Journal of Information \& Knowledge Management 5(3). 193-210.

Roepstorff, Lars, Agneta Egenvall, Marie Rhodin, Anna Byström, C. Johnston, P. R. Van Weeren \& M. Weishaupt. 2009. Kinetics and kinematics of the horse comparing left and right rising trot. Equine Veterinary Journal 41(3). 292-296.

Senft, Gunter. 2001. Frames of spatial reference in Kilivila. Studies in Language 25(3). 521-555.

Stewart, Nigel. 1998. Re-languaging the body: Phenomenological description and the dance image. Performance Research 3(2). 42-53.

Talmy, Leonard. 2000. Toward a cognitive semantics. Cambridge, MA: MIT Press.

Taylor, Holly A. \& Tversky Barbara. 1992. Spatial mental models derived from survey and route descriptions. Journal of Memory \& Language 31. 261-292.

Tenbrink, Thora. 2007. Space, time, and the use of language: An investigation of relationships. Berlin: Mouton de Gruyter.

Tenbrink, Thora. 2011. Reference frames of space and time in language. Journal of Pragmatics 43(3). 704-722.

Tenbrink, Thora. 2012. Relevance in spatial navigation and communication. In Cyrill Stachniss, Kerstin Schill \& David Uttal (eds.), Spatial Cognition 2012, LNAI 7463, 358-377. Berlin: Springer.

Tenbrink, Thora, Kenny R. Coventry \& Andonova Elena. 2011. Spatial strategies in the description of complex configurations. Discourse Processes 48. 237-266. 
Tenbrink, Thora \& Frank Dylla. 2017. Sailing: Cognition, action, communication. Journal of Spatial Information Science $15.3-33$. Tversky, Barbara, Paul Lee \& Mainwaring Scott. 1999. Why do speakers mix perspectives? Spatial Cognition \& Computation 1(4). 399-412.

Tyler, Andrea \& Vyvyan Evans. 2003. The semantics of English prepositions: Spatial scenes, embodied meaning, and cognition. Cambridge: Cambridge University Press.

van der Zee, Emile \& Rik Eshuis. 2003. Directions from shape: How spatial features determine reference axis categorization. In Emile van der Zee \& Jon Slack (eds.), Representing direction in language and space, 209-225. Oxford: Oxford University Press. Wigman, Mary. 1966. The language of dance. Middletown: Wesleyan University Press. 